\title{
Control of the Ultrafast Photoinduced Magnetization across the Morin Transition in $\mathrm{DyFeO}_{3}$
}

\author{
D. Afanasiev, ${ }^{1, *}$ B. A. Ivanov, ${ }^{2,3}$ A. Kirilyuk, ${ }^{1}$ Th. Rasing, ${ }^{1}$ R. V. Pisarev, ${ }^{4}$ and A. V. Kimel ${ }^{1}$ \\ ${ }^{1}$ Radboud University, Institute for Molecules and Materials, 6525 AJ Nijmegen, The Netherlands \\ ${ }^{2}$ Institute of Magnetism, National Academy of Sciences, 03142 Kiev, Ukraine \\ ${ }^{3}$ Taras Shevchenko National University of Kiev, 01601 Kiev, Ukraine \\ ${ }^{4}$ Ioffe Physical-Technical Institute RAS, 194021 St. Petersburg, Russia
}

(Received 6 October 2015; published 3 March 2016)

\begin{abstract}
Excitation of the collinear compensated antiferromagnet $\mathrm{DyFeO}_{3}$ with a single $60 \mathrm{fs}$ laser pulse triggers a phase transition across the Morin point into a noncollinear spin state with a net magnetization. Timeresolved imaging of the magnetization dynamics of this process reveals that the pulse first excites the spin oscillations upon damping of which the noncollinear spin state emerges. The sign of the photoinduced magnetization is defined by the relative orientation of the pump polarization and the direction of the antiferromagnetic vector in the initial collinear spin state.
\end{abstract}

DOI: 10.1103/PhysRevLett.116.097401

Photoinduced nonequilibrium phase transitions triggered by femtosecond laser pulses and their kinetics are subjects of intense and multidisciplinary research [1-5]. In magnetism this is a particularly important problem, the understanding of which might be essential for future progress in information processing technology. The ability to visualize the magnetization dynamics in a laser excited area with femtosecond temporal resolution was shown to be crucial for understanding the physics of photo-induced magnetic phase transitions and resulted into discovery of several mechanisms of optical control of magnetism [6-8].

The question of how femtosecond laser excitation can result in a subpicosecond emergence of the net magnetization remains very controversial [9-14]. A phase with spontaneous magnetization has at least two degenerate states corresponding to two opposite magnetization directions. If laser excitation deterministically induces a net magnetization, it means that the degeneracy is broken. In thermodynamics the speed with which the magnetization emerges is defined by the energy of interaction that breaks this degeneracy. Subpicosecond emergence of the magnetization in antiferromagnetic FeRh and manganites was reported in Refs. [9-14]. Magnetic fields in these experiments were very small and the Zeeman splitting could not explain spin dynamics faster than 10 ps. Revealing the mechanisms responsible for the emergence of the photoinduced magnetization is one of the main challenges in ultrafast magnetism.

In this Letter we report about the ultrafast kinetics of the photoinduced first-order Morin phase transition from a compensated collinear to a noncollinear antiferromagnetic state in $\mathrm{DyFeO}_{3}$. Visualizing the emerging magnetization with femtosecond temporal and micrometer spatial resolution, we reveal three main stages of the photoinduced phase transition: (i) a sub-100 fs laser pulse generates an effective magnetic field due to the optomagnetic effect [15] and launches spin oscillations corresponding to the soft mode of the phase transition, (ii) the photoinduced magnetization sets in within the first half of the oscillations period (2.5 ps), (iii) the magnetization grows on a scale of $100 \mathrm{ps}$. It is revealed that the phase of the oscillation and the direction of the photoinduced magnetization are defined by the light polarization and the direction of the antiferromagnetic vector in the initial state. These findings are corroborated by a phenomenological description, that suggests a coupling of the antiferromagnetic vector with the electric field of light.

Dysprosium orthoferrite $\left(\mathrm{DyFeO}_{3}\right)$ crystallizes in an orthorhombic structure (space group is $D_{2 h}^{16}-\mathrm{Pbnm}$ ). $\mathrm{Fe}^{3+}$ spins are coupled antiferromagnetically and retain their order up to $645 \mathrm{~K}$. At $T_{\mathrm{M}} \simeq 39 \mathrm{~K}$, called the Morin point, the orthoferrite exhibits a reorientational magnetic phase transition [16-18]. Below $T_{M}$ the spins are in the compensated collinear antiferromagnetic $\Gamma_{1}$ phase. The magnetic moments of the two sublattices $\left(\mathbf{M}_{1}, \mathbf{M}_{2}\right)$ and the antiferromagnetic vector $\mathbf{L}=\mathbf{M}_{2}-\mathbf{M}_{1}$ are along the $y$ axis and there is no net magnetization $\mathbf{M}=\mathbf{M}_{1}+\mathbf{M}_{2}=0$. Without an external stimulus the crystal splits into 180degree antiferromagnetic domains. Above $T_{M}$ the spins are in the $\Gamma_{4}$ phase. The magnetic moments of the sublattices and the antiferromagnetic vector $\mathbf{L}$ are then aligned along the $x$ axis. Becasue of the Dzyaloshinskii-Moriya interaction the spins of the two sublattices acquire a canting over an angle of about 0.5 degrees. This gives rise to a small spontaneous magnetization $\mathbf{M}=\mathbf{M}_{1}+\mathbf{M}_{2} \neq 0$ along the $z$ axis. In contrast to many spin reorientations in orthoferrites, which take place through two second-order phase transitions [19], the Morin transition in $\mathrm{DyFeO}_{3}$ is of the first order. Thus it is accompanied by a sudden emergence of a net magnetization (see Supplemental Material [20]). The phase transition at $T_{M}$ is explained as a result of the 
thermally induced repopulation of low electronic states of $\mathrm{Dy}^{3+}$ ions which affects the interplay between two contributions to the magnetic anisotropy experienced by the $\mathrm{Fe}^{3+}$ spins [19].

In order to trigger the Morin transition on the ultrafast time scale, the sample was excited by a 60 fs laser pulse at a central wavelength of $800 \mathrm{~nm}$. At this wavelength the absorption coefficient of the material is about $80 \mathrm{~cm}^{-1}$ [21]. The pump pulse was at normal incidence. The beam had a Gaussian spatial profile, and was focused into a spot with a full width at half maximum (FWHM) of $40 \mu \mathrm{m}$. The repetition rate of the pump pulse was brought down to $2 \mathrm{~Hz}$. It allowed for a substantial increase of the pump fluence in comparison with previous experiments $[8,22]$. The CCD camera detecting the probe was triggered by the pump to obtain single-shot images. At the fluence of $0.1 \mathrm{~J} / \mathrm{cm}^{2}$ each of the pulses heats the sample up by around $30 \mathrm{~K}$. Afterwards the sample cools down on a ms time scale to the initial temperature.

For the study we chose a sample cut perpendicular to the $z$ crystallographic axis $\left(z-\mathrm{DyFeO}_{3}\right)$. A typical pattern of dark-grey and light-grey areas, representing magnetooptical images of the 180-degree antiferromagnetic domains $\mathbf{L}+$ and $\mathbf{L}-$ in the low temperature $\Gamma_{1}$ phase, is shown in Fig. 1. Such a contrast between the domains is achieved due to contributions to the symmetric part of the dielectric permittivity tensor proportional to the product of the antiferromagnetic vector and the applied mechanical strain [23]. In our experiments the stress was applied in the [110] crystallographic direction. The magnetooptical image of the much smaller labyrinth domains in the high temperature $\Gamma_{4}$ phase is shown in Fig. 1(b). The white and black areas correspond to the domains in which $M_{z}$ is parallel (plus) and antiparallel (minus) with respect to the $z$-axis directions, respectively.

The single pump pulse excitation of the $z-\mathrm{DyFeO}_{3}$ initiates well-pronounced magnetic changes [Fig. 1(c)]. It is seen that pumping the dark-grey $+L_{y}$ areas produces a black domain, whereas pumping the light-grey $-L_{y}$ areas produces a white domain. Pumping in such a way that the domain wall is right in the middle of the laser spot produces two domains with their magnetization depending on the $\pm L_{y}$ orientation. We assign the photoinduced black and white areas to domains with a photoinduced $\pm M_{z}$ component, respectively. Hence, this experiment reveals that the orientation of the photoinduced magnetization in $z-\mathrm{DyFeO}_{3}$ is unambiguously defined by the sign of the antiferromagnetic $L_{y}$ component in the initial state.

The dynamics of the photoinduced magnetization is shown in Fig. 2(a). The emergence as well as the growth of the induced magnetization inherent to the hightemperature $\Gamma_{4}$ phase are clearly seen. Within the first $20 \mathrm{ps,}$ however, the observation of magnetic dynamics is slightly hampered by photoinduced transmission change and its dynamics [24]. Taking a cross section of the spot, we obtain

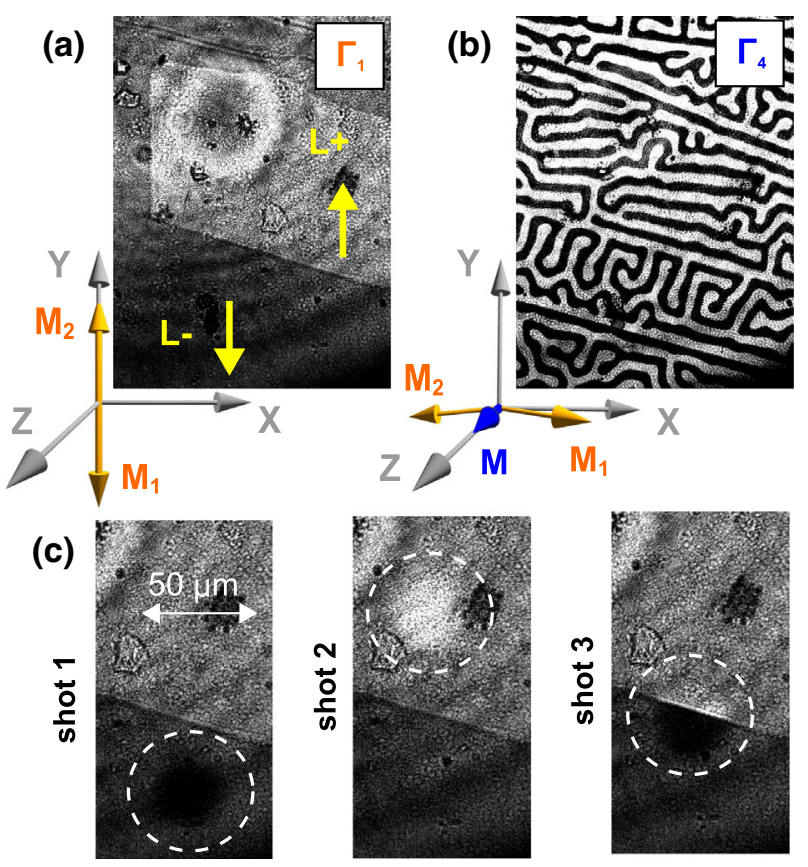

FIG. 1. (a) Magnetooptical images of the two types of antiferromagnetic domains in the low-temperature $\Gamma_{1}$ phase of the $z-\mathrm{DyFeO}_{3}$ sample. The bias temperature of the sample is $20 \mathrm{~K}$. (b) Magnetooptical image of the magnetic domain pattern in the high-temperature $\Gamma_{4}$ phase. The bias temperature of the sample is $50 \mathrm{~K}$. (c) Images of the domains in $z-\mathrm{DyFeO}_{3}$ taken $100 \mathrm{ps}$ after excitation with a single 60 fs pump pulse. Areas exposed to the pulsed excitation are marked by dashed circles. "shot 1 " forms a black domain having $-M_{z}$ magnetization in the dark-grey area, corresponding to $\mathbf{L}-$. "shot 2 " forms a white domain with the $+M_{z}$ magnetization in the light-grey area, $\mathbf{L}+$. "shot 3 " affects both antiferromagnetic domains and induces a magnetization the sign of which is defined by the direction of $\mathbf{L}$.

the dynamics of the photoinduced magnetization across the laser excited area [Fig. 2(b)]. Regarding the Gaussian spatial distribution of the laser intensity, the obtained twodimensional images reveal how the dynamics depends on the laser fluence, indicating that the photoinduced magnetization emerges exponentially but only in the areas where the fluence is above $0.25 \mathrm{~J} / \mathrm{cm}^{2}$.

Coherent oscillations of the magnetization exist across the whole photoexcited area. The frequency of the oscillations $f_{a}=170 \mathrm{GHz}$ does not depend on the fluence, but the damping is clearly larger at higher fluences [Fig. 2(c)]. Performing the measurements at different temperatures we found that the temperature behavior of the frequency $f_{a}$ strictly follows the frequency of the soft mode of the Morin transition [25]. It is associated with spin motion in the (001) plane, such that finite $L_{x}$ and $M_{z}$ components appear. Although the damping ratio of the spin oscillations and the characteristic rise time of the transition are functions of the intensity of the laser excitation, the product of these two quantities remains nearly constant. These observations show that the spin oscillations at the frequency of the soft 


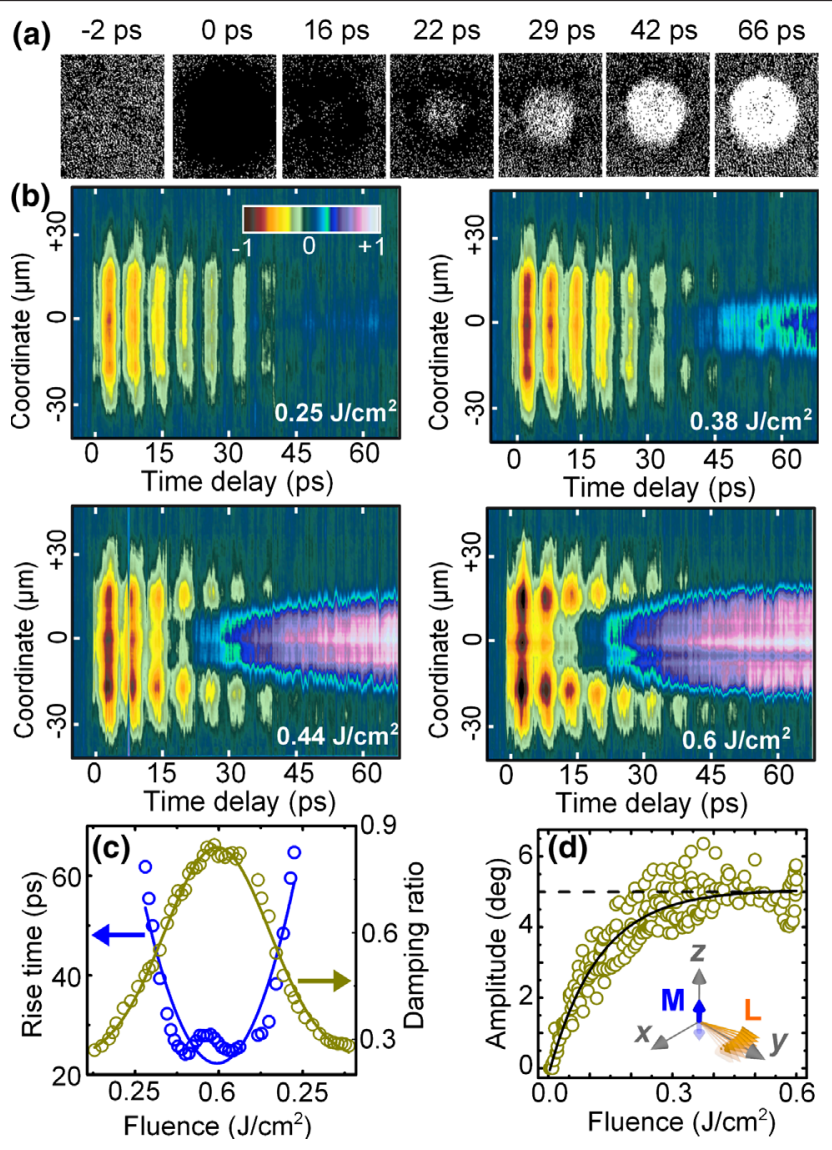

FIG. 2. (a) Time-resolved magnetooptical images of the photoinduced dynamics in $z-\mathrm{DyFeO}_{3}$. The images are obtained by taking the difference between the images at the given positive delay and those at negative delay. The bias sample temperature is set to $23 \mathrm{~K}$. (b) The magnetization dynamics in a cross section of the laser-excited area for the different excitation fluences. The left vertical axis shows the distance from the center of the laser excited area. Each figure is defined by the fluence at the center of the pump spot. The color code represents the measured value of the magnetooptical signal and is the same for all pictures in the panel. (c) The distribution of the damping ratio of the spin oscillations and rise time of the photoinduced magnetization extracted in the cross section. Coordinates are replaced with values of the local fluence. Solid lines are the Gaussian fits of the dependencies with FWHM of the pump beam. (d) Amplitude of the pump-induced antiferromagnetic spin oscillations in the $\Gamma_{1}$ phase for different pump fluences. The value of the amplitude was obtained by comparing the time-resolved magnetooptical signal with the Faraday rotation in the $\Gamma_{4}$ phase. The solid line is a fit with a single exponent. The inset is a schematic representations of the spin oscillations.

mode and the Morin transition are mutually correlated. The amplitude of the soft mode saturates for the pump fluence of about $0.3 \mathrm{~J} / \mathrm{cm}^{2}$ [Fig. 2(d)] which is very close to the fluence threshold required for the emergence of the photoinduced magnetization. At this fluence the amplitude of the spin oscillations is estimated to be about $5^{\circ}$. This value of the amplitude is considered to be a critical angle $\theta_{\mathrm{cr}}$, which spins have to deviate on to promote the Morin transition.
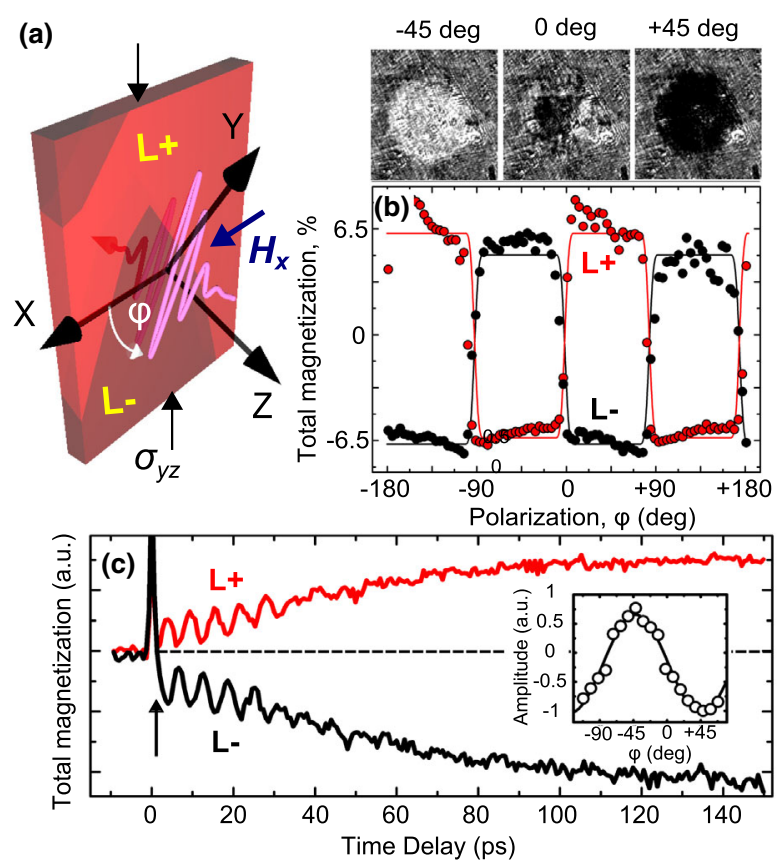

FIG. 3. (a) The geometry of the experiment with a single crystal cut perpendicular to the optical axis: the $o-\mathrm{DyFeO}_{3}$. The external magnetic field $H_{x}$ and applied stress $\sigma_{y z}$ are depicted on the picture. The pump is linearly polarized. (b) The total magnetization integrated over the spot as a function of the angle $\varphi$ between the pump polarization and the $x$ axis for two antiferromagnetic domains with opposite signs of the $L_{y}$ component. The value is shown by a percentage of the value of the static Faraday rotation in the $\Gamma_{4}$ phase. The solid lines are square wave fits. (c) The dynamics of the total magnetization for two antiferromagnetic domains obtained by applying magnetic field $H_{x}= \pm 1.7 \mathrm{kOe}$. Each curve is obtained as a difference between two experiments performed for two polarizations of the pump $\varphi= \pm 45$ degrees. The inset shows the amplitude of the spin oscillations as a function of the angle $\varphi$.

To reveal the mechanism responsible for the optical excitation of spins, one needs to study the laser-induced dynamics as a function of the pump pulse polarization $[26,27]$. However, the $z-\mathrm{DyFeO}_{3}$ crystal is characterized by a very strong linear birefringence [28]. In order to reveal the genuine polarization dependence of the mechanism responsible for the optical excitation of spins, we took a sample cut perpendicular to the optical axis $\left(o-\mathrm{DyFeO}_{3}\right)$. To bring the sample into a single antiferromagnetic domain state, a stress $\sigma_{y z}$ applied in the (100) plane was combined with a moderate bias magnetic field $H_{x}$ [29]. We studied laserinduced magnetization as a function of the pump pulse polarization in both antiferromagnetic domains. The geometry of the experiment and the experimental results are shown in Fig. 3.

It is clearly seen that the magnetization state long after the excitation strongly depends on the azimuthal orientation of the linear polarization of the pump [Fig. 3(b)]. Taking the integral over the photoexcited area for the 
images measured at $2.6 \mathrm{~ns}$ after the excitation, we obtain a measure of the total photoinduced magnetization. Figure 3 shows the azimuthal dependence of this integral as a function of the angle $\varphi$ between the pump polarization and the $x$ axis. The dependence has nearly $180^{\circ}$ steplike periodicity. In agreement with the discussed above effect in the $z-\mathrm{DyFeO}_{3}$ sample, a reversal of $\mathbf{L}$ changes the sign of the photoinduced magnetization. Hence, the plus or minus orientation of the induced magnetization is defined not only by the orientation of the antiferromagnetic vector $\mathbf{L}$ in the initial collinear phase $\Gamma_{1}$ but also by the polarization. These findings can be summarized by an expression: $\operatorname{sgn}\left(M_{z}\right)=\operatorname{sgn}\left(L_{y}\right) \operatorname{sgn}\left(\mathcal{E}_{x} \mathcal{E}_{y}^{*}+\right.$ c.c. $)$, where $\mathcal{E}_{x}(t)$ and $\mathcal{E}_{y}(t)$ are projections of the electric field of the pump light on crystallographic axes.

Essential theoretical insight into the physics of the ultrafast dynamics of antiferromagnets can be obtained using the sigma-model equation, derived from the free energy of light-matter interaction $W_{\operatorname{lm}}$ [30]. The lowest order term allowed by the magnetic symmetry of the soft mode in the $\Gamma_{1}$ phase for linearly polarized light is

$$
W_{\mathrm{lm}}=\left(\mathcal{A} L_{x}+\mathcal{B} M_{z}\right) L_{y}\left(\mathcal{E}_{x} \mathcal{E}_{y}^{*}+\text { c.c. }\right) \text {. }
$$

Here $\mathcal{A}$ and $\mathcal{B}$ are phenomenological parameters. For the dynamics of the soft mode the sigma-model equation reads

$$
\frac{\partial^{2} L_{x}}{\partial t^{2}}+2 \lambda \frac{\partial L_{x}}{\partial t}+\omega_{a}^{2} L_{x}=\begin{array}{r}
-\gamma^{2} H_{\mathrm{ex}} \mathcal{A}|L| L_{y}\left(\mathcal{E}_{x} \mathcal{E}_{y}^{*}+\text { c.c. }\right) \\
+\mathcal{B} \gamma L_{y}^{2} \frac{d}{d t}\left(\mathcal{E}_{x} \mathcal{E}_{y}^{*}+\text { c.c. }\right),
\end{array}
$$

where $H_{\mathrm{ex}}$ is the exchange field, $\gamma$ is the gyromagnetic ratio, $\lambda$ is a phenomenological damping parameter, $\omega_{a}=2 \pi f_{a}$.

After the standard substitution, $\left(\mathcal{E}_{x} \mathcal{E}_{y}^{*}+\right.$ c.c. $) \rightarrow$ $I_{0} \delta(t) \sin 2 \varphi$, where $I_{0}$ is the pulse fluence and $\delta(t)$ is the delta function one can see that the first term on the right-hand side of Eq. (2) creates an initial speed of spin rotation. Thus it initiates the inertial spin dynamics in the antiferromagnet [22]. The spin oscillations can be launched via this mechanism, which can be seen as the inverse Cotton-Mouton effect [26]. This excitation can also lead to a homogeneous rotation of spins from the initial collinear phase $\Gamma_{1}$ to one of the minima in noncollinear $\Gamma_{4}$ phase corresponding to the opposite directions of the magnetic moment $M_{z}=$ $H_{D} L_{x} / H_{\mathrm{ex}}, H_{D}$ is the Dzyaloshinskii field.

This picture is confirmed by the experimentally observed dynamics of the total photoinduced magnetization in $o-\mathrm{DyFeO}_{3}$ [Fig. 3(c)]. The measurements were performed for the two domains with opposite orientation of the antiferromagnetic vector $\mathbf{L}$. It is seen that the laser pulse induces a net magnetization $M_{z}$ already within the first $2.5 \mathrm{ps}$ after the excitation. It corresponds to the half of the period of the spin oscillations. The damped spin oscillations take place on the background of the emergent magnetization. Note, that at the much longer delay of $2.6 \mathrm{~ns}$ the photoinduced magnetization is still much lower than that in the thermodynamic equilibrium in the $\Gamma_{4}$ phase. A larger magnetization builds up on a longer time scale.

The whole set of experimental data is drastically different from that in the case of the photoinduced second-order phase transition [8]. In the present case, the transition is more robust since the direction of the induced magnetization is fully defined by the light polarization and the direction of the antiferromagnetic vector. Unlike Ref. [8], here we did not reveal any sensitivity of the magnetization direction in the photoinduced state to the initial temperature and/or the laser fluence. We relate these observations to peculiarities of the free energy landscape in the vicinity of first-order phase transitions. In $\mathrm{DyFeO}_{3}$ just below $T_{M}$ one can distinguish potential minima corresponding to the stable $\Gamma_{1}$ phase and the metastable $\Gamma_{4}$ phase. The temperature dependence of the square of the frequency of the soft mode shows that the interval in which the phases coexist (from $T_{1}$ to $T_{2}$ ) should be quite wide (see Fig. 4(a), for more details see Supplemental Material [20]). Nevertheless, the temperature hysteresis has never been observed experimentally for the Morin transition in $\mathrm{DyFeO}_{3}[19,23]$. These facts reveal that the Morin transition is very susceptible to fluctuations and nuclei of a metastable phase are easily formed in the vicinity of the first-order phase transition. As a result of fluctuations, the temperature of the Morin transition can be locally reduced together with the potential barrier between the metastable and stable states. In such areas the potential barrier which separates the minima is reduced [see Fig. 4(b)]. The ultrashort laser pulse kicks the spins and launches spin oscillations of the soft mode. As soon as spin deviation

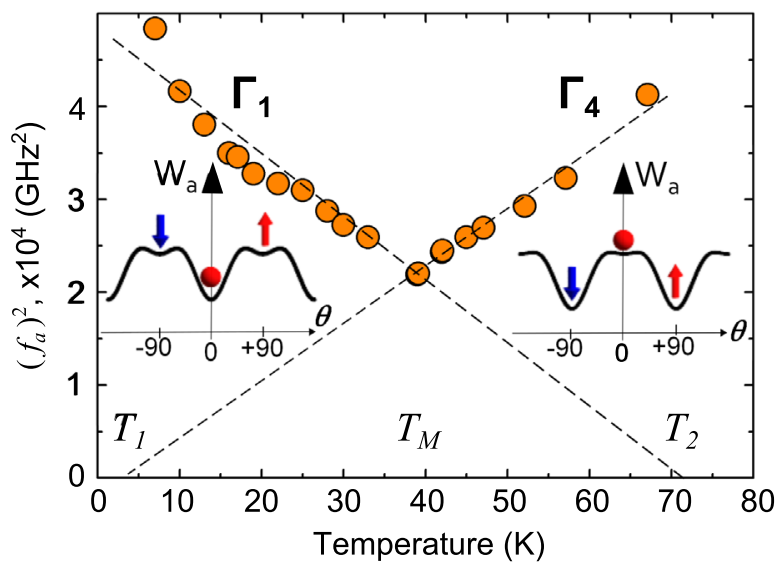

FIG. 4. (a) The square of the frequency $f_{a}$ of the photoinduced oscillations for different temperatures in the vicinity of the Morin point. Dashed lines are linear fits of frequencies extended to the intersection with the temperature axis. The intersection points $T_{1}$ and $T_{2}$ define the region where thermodynamically phases $\Gamma_{1}$ and $\Gamma_{4}$ may coexist. Insets show the magnetic anisotropy potential $W_{a}$ of the first-order Morin phase transition as a function of angle $\theta$, the spins form with the $y$ axis for two temperatures $T_{1}<T<T_{M}$ and $T_{M}<T<T_{2}$. 
reaches the critical angle $\theta_{\mathrm{cr}}$ the first nuclei of the $\Gamma_{4}$ phase are created. The direction of the magnetization in these nuclei is given by the direction of the initial kick of spins. If the same pump pulse causes laser-induced heating across the $T_{M}$, the $\Gamma_{4}$ phase becomes stable over the whole laserexcited spot. This fact promotes a gradual growth of the already created $\Gamma_{4}$ nuclei, depletion of the $\Gamma_{1}$ phase, and damping of the spin oscillations.

To summarize, our study reveals the ultrafast kinetics of the photoinduced emergence of magnetization in a collinear compensated antiferromagnet and demonstrates the decisive role of the antiferromagnetic vector, the role of which in photoinduced phase transitions had been previously ignored. We note that, in fact, this Letter describes how and how fast the Dzyaloshinskii-Morya interaction introduces noncollinearities in the spin structure of a magnet. Therefore, the approach reported here offers intriguing opportunities for the investigation of such heavily debated topics of condensed matter physics as the emergence of helicoidal spin textures, skyrmions, and multiferroicity.

The authors thank R. Mikhaylovskiy and A. Zvezdin for fruitful discussions, T. Toonen and A. van Etteger for technical support, and A. M. Balbashov for providing the $o-\mathrm{DyFeO}_{3}$ sample. This work was partially supported by The Netherlands Organization for Scientific Research (NWO), the Foundation for Fundamental Research on Matter (FOM), the EU Seventh Framework Program (FP7/2007-2013) Grants No. NMP3-LA-2010-246102 (IFOX), No. 280555 (Go-Fast), the European Research Council (FP7/2007-2013)/ERC Grant Agreements No. 257280 (Femtomagnetism) and No. 339813 (Exchange). R. V. P. acknowledges partial support by the Russian Projects No. 14.B25.31.0025 (Ministry of Education and Science) and No. 15-02-14222 (RFBR).

*d.afanasiev@science.ru.nl

[1] M. Chollet et al., Science 307, 86 (2005).

[2] A. Cavalleri, C. Tóth, C. W. Siders, J. A. Squier, F. Ráksi, P. Forget, and J. C. Kieffer, Phys. Rev. Lett. 87, 237401 (2001).

[3] P. Beaud, A. Caviezel, S. O. Mariager, L. Rettig, G. Ingold, C. Dornes, S.-W. Huang, J. A. Johnson, M. Radovic, T. Huber, T. Kubacka, A. Ferrer, H. T. Lemke, M. Chollet, D. Zhu, J. M. Glownia, M. Sikorski, A. Robert, H. Wadati, M. Nakamura, M. Kawasaki, Y. Tokura, S. L. Johnson, and U. Staub, Nat. Mater. 13, 923 (2014).

[4] H. Ichikawa, S. Nozawa, T. Sato, A. Tomita, K. Ichiyanagi, M. Chollet, L. Guerin, N. Dean, A. Cavalleri, S.-i. Adachi, T.-h. Arima, H. Sawa, Y. Ogimoto, M. Nakamura, R. Tamaki, K. Miyano, and S.-y. Koshihara, Nat. Mater. 10, 101 (2011).

[5] S. L. Johnson, R. A. de Souza, U. Staub, P. Beaud, E. Möhr-Vorobeva, G. Ingold, A. Caviezel, V. Scagnoli, W. F. Schlotter, J. J. Turner, O. Krupin, W.-S. Lee, Y.-D. Chuang, L. Patthey, R. G. Moore, D. Lu, M. Yi, P. S. Kirchmann, M. Trigo, P. Denes, D. Doering, Z. Hussain, Z.-X. Shen,
D. Prabhakaran, and A. T. Boothroyd, Phys. Rev. Lett. 108, 037203 (2012).

[6] C. von Korff Schmising et al., Phys. Rev. Lett. 112, 217203 (2014).

[7] C. Graves et al., Nat. Mater. 12, 293 (2013).

[8] J. A. de Jong, I. Razdolski, A. M. Kalashnikova, R. V. Pisarev, A. M. Balbashov, A. Kirilyuk, T. Rasing, and A. V. Kimel, Phys. Rev. Lett. 108, 157601 (2012).

[9] G. Ju, J. Hohlfeld, B. Bergman, R. J. M. van de Veerdonk, O. N. Mryasov, J.-Y. Kim, X. Wu, D. Weller, and B. Koopmans, Phys. Rev. Lett. 93, 197403 (2004).

[10] B. Bergman, G. Ju, J. Hohlfeld, R. J. M. van de Veerdonk, J.-Y. Kim, X. Wu, D. Weller, and B. Koopmans, Phys. Rev. B 73, 060407 (2006).

[11] S. O. Mariager, F. Pressacco, G. Ingold, A. Caviezel, E. Möhr-Vorobeva, P. Beaud, S. L. Johnson, C. J. Milne, E. Mancini, S. Moyerman, E. E. Fullerton, R. Feidenhans'l, C. H. Back, and C. Quitmann, Phys. Rev. Lett. 108, 087201 (2012).

[12] K. Miyasaka, M. Nakamura, Y. Ogimoto, H. Tamaru, and K. Miyano, Phys. Rev. B 74, 012401 (2006).

[13] M. Matsubara, Y. Okimoto, T. Ogasawara, Y. Tomioka, H. Okamoto, and Y. Tokura, Phys. Rev. Lett. 99, 207401 (2007).

[14] T. Li, A. Patz, L. Mouchliadis, J. Yan, T. A. Lograsso, I. E. Perakis, and J. Wang, Nature (London) 496, 69 (2013).

[15] A. V. Kimel, A. Kirilyuk, and T. Rasing, Laser Photonics Rev. 1, 275 (2007).

[16] A. Maziewski and R. Szymczak, J. Phys. D 10, L37 (1977).

[17] F. Hong, Z. Cheng, H. Zhao, H. Kimura, and X. Wang, Appl. Phys. Lett. 99, 092502 (2011).

[18] W. Zhao, S. Cao, R. Huang, Y. Cao, K. Xu, B. Kang, J. Zhang, and W. Ren, Phys. Rev. B 91, 104425 (2015).

[19] K. P. Belov, A. K. Zvezdin, A. M. Kadomtseva, and R. Levitin, Physics Uspekhi 19, 574 (1976).

[20] See Supplemental Material at http://link.aps.org/ supplemental/10.1103/PhysRevLett.116.097401 for details about measurements of the Faraday rotation as a function of temperature, conversion of the spatial coordinates into the local fluence, data fitting procedure and determination of the region where the phases coexistence occurs.

[21] D. L. Wood, J. P. Remeika, and E. D. Kolb, J. Appl. Phys. 41, 5315 (1970).

[22] A. V. Kimel, B. A. Ivanov, R. V. Pisarev, P. A. Usachev, A. Kirilyuk, and T. Rasing, Nat. Phys. 5, 727 (2009).

[23] V. V. Eremenko and N. Kharchenko, Phys. Rep. 155, 379 (1987).

[24] A. V. Kimel, R. V. Pisarev, J. Hohlfeld, and T. Rasing, Phys. Rev. Lett. 89, 287401 (2002).

[25] A. Balbashov, A. Volkov, S. Lebedev, A. Mukhin, and A. Prokhorov, Zh. Eksp. Teor. Fiz. 88, 974 (1985).

[26] A. M. Kalashnikova, A. V. Kimel, R. V. Pisarev, V. N. Gridnev, A. Kirilyuk, and T. Rasing, Phys. Rev. Lett. 99, 167205 (2007).

[27] R. Iida, T. Satoh, T. Shimura, K. Kuroda, B. A. Ivanov, Y. Tokunaga, and Y. Tokura, Phys. Rev. B 84, 064402 (2011).

[28] V. G. Baryakhtar, B. A. Ivanov, and M. V. Chetkin, Physics Uspekhi 28, 563 (1985).

[29] S. L. Gnatchenko, A. B. Chizhik, V. A. Bedarev, and N. F. Kharchenko, Low Temp. Phys. 21, 736 (1995).

[30] B. A. Ivanov, Low Temp. Phys. 40, 91 (2014). 